\section{Índice de Cobertura Assistencial da Rede de Atenção Psicossocial (iRAPS) como ferramenta de análise crítica da reforma psiquiátrica brasileira}

\author{
Healthcare Coverage Index in the Psychosocial \\ Care Network (iRAPS) as a tool for critical analysis \\ of the Brazilian psychiatric reform
}

Índice de Cobertura Asistencial de la Red de Atención Psicosocial (iRAPS) como herramienta de análisis-crítico de la reforma psiquiátrica brasileña

\author{
Cristofthe Jonath Fernandes 1,2 \\ Aluísio Ferreira de Lima 1 \\ Pedro Renan Santos de Oliveira 3 \\ Walberto Silva dos Santos 1
}

\title{
Resumo
}

Passados quase vinte anos de implantação da reforma psiquiátrica no Brasil, o país conta com uma assistência à saúde mental híbrida, na qual os serviços asilares são parte da própria rede que deveria substituí-los. Este estudo buscou analisar criticamente a oferta dos serviços de assistência à saúde mental no país, no intuito de identificar se efetivamente decorre um processo de substituição dos serviços de ênfase asilar pelos comunitários. Para alcançar o objetivo, foi realizado um estudo longitudinal retrospectivo da oferta de serviços de assistência à saúde mental no Brasil, no período de 2008 a 2017, com a elaboração de uma base de dados única com informações dos 5.570 municípios. Como ferramenta auxiliar da análise crítica da reforma psiquiátrica, foi construído o Índice de Cobertura Assistencial da Rede de Atenção Psicossocial (iRAPS), considerando-se a parametrização legal dos serviços. Foi verificado o avanço da oferta dos serviços comunitários e a redução dos asilares. Porém, com o iRAPS identificou-se que 77\% da população brasileira habitam localidades com cobertura de serviços comunitários baixa ou inexistente. As pequenas cidades, que concentram 16\% da população nacional, foram as que mais impulsionaram o avanço do iRAPS em nível nacional. Apenas 439 cidades, 7,9\% do total, apresentam cobertura assistencial total da Rede de Atenção Psicossocial (RAPS), expressando apenas 6,69\% da populacional nacional. As metrópoles, que concentrarem 46\% da população brasileira, não apresentaram avanço na cobertura de serviços comunitários. A análise da oferta de serviços de saúde mental com base no iRAPS permiti-nos afirmar que o avanço dos serviços comunitários ocorreu de forma não homogênea entre as localidades.

Psicologia Social; Saúde Mental; Cobertura de Serviços de Saúde;

Desinstitucionalização; Hospitais Psiquiátricos

\author{
Correspondência \\ C. J. Fernandes \\ Universidade Federal do Ceará. \\ Av. da Universidade 2762, Fortaleza, CE 60020-180, Brasil. \\ cjonath@gmail.com \\ 1 Universidade Federal do Ceará, Fortaleza, Brasil. \\ 2 Faculdade de Psicologia e Ciências da Educação, Universidade \\ do Porto, Porto, Portugal. \\ ${ }_{3}^{3}$ Centro Universitário Christus, Fortaleza, Brasil.
}




\section{Introdução}

Os sofrimentos relacionados à saúde mental representam um problema mundial. Não por acaso, seguindo as recomendações da Organização Mundial da Saúde (OMS), nos últimos anos foram elaboradas políticas nacionais específicas para esse campo em todas as partes do mundo. Na América do Sul, cinco países (Argentina, Brasil, Equador, Paraguai e Uruguai) têm algum documento que explicita uma política de saúde mental. Em quase todos os casos, as implementações dessas políticas são recentes, posteriores ao ano de 20021,2 .

No Brasil, presenciamos atualmente um quarto momento da política de saúde mental, iniciado de maneira mais organizado a partir do final dos anos de 1970. Impulsionada pela redemocratização do país e pela Reforma Sanitária, que originou o Sistema Único de Saúde (SUS) e possibilitou a entrada de militantes do movimento antimanicomial nos aparelhos de Estado, inaugurou-se o que foi nomeado como reforma psiquiátrica. Dentre o arcabouço jurídico-legal que constituiu essa reforma, destacouse a Lei no 10.216/2001 ${ }^{3}$ que, conforme afirmou o próprio Estado brasileiro, em documento enviado à Comissão Interamericana de Direitos Humanos, veio responder "a antigos reclamos do movimento antimanicomial" 4, transformando o que era uma proposição alternativa à política de saúde mental desenvolvida, em uma nova política de Estado.

Assim, consideraremos neste estudo que a Lei no 10.216/2001 buscou formalizar a principal reivindicação do movimento antimanicomial, que consiste na construção de "uma sociedade sem manicômios". A fundamentação teórico-metodológica da reforma brasileira é notoriamente alinhada à psiquiatria democrática italiana, que insistia na importância da criação de serviços totalmente substitutivos ao hospital psiquiátrico 5 . O inciso IX do artigo terceiro da referida lei expressa o redirecionamento para uma assistência à saúde mental comunitária, ao afirmar que a pessoa deve "ser tratada, preferencialmente, em serviços comunitários de saúde mental". Serviços esses que se caracterizam por prestarem assistência à saúde mental de forma que o sujeito perpetue seus laços sociais, se contrapondo aos serviços ofertados pela lógica asilar, que até então era protagonista na assistência à saúde mental do país e se caracterizava pelo internamento de longas durações em instituições fechadas, excluindo socialmente os sujeitos atendidos.

Em menos de uma década, considerando-se o ano de 2008 como exemplo, o Brasil contava com 891 serviços substitutivos comunitários e reduzia o número de leitos nos hospitais psiquiátricos 2 , tratando-se da evidência mais imediata dos avanços constatada no campo da denominada reforma psiquiátrica.

No ano de 2011 foi emitida a Portaria no 3.088 do Ministério da Saúde 6 que, organizando o trabalho conjunto dos diversos serviços de atenção à saúde mental de base comunitária, instituiu a Rede de Atenção Psicossocial (RAPS). Essa rede nasceu formada pelos seguintes componentes: (i) Atenção Básica em Saúde; (ii) Atenção Psicossocial Especializada; (iii) Atenção de Urgência e Emergência; (iv) Atenção Residencial de Caráter Transitório; (v) Atenção Hospitalar; (vi) Estratégias de Desinstitucionalização; e, por fim, (vii) Reabilitação Psicossocial. Cada um desses componentes, por sua vez, detém um conjunto específico de pontos de atenção e linhas de cuidado.

Em data recente, ao final de 2017, o Ministério da Saúde emitiu a Portaria no 3.5887 que deu nova formatação ao componente hospitalar da RAPS e gerou polêmica entre aqueles que defendem a reforma psiquiátrica, no que diz respeito à manutenção do seu próprio caráter. Isso porque o componente hospitalar da RAPS, além dos leitos psiquiátricos em hospital geral (LHG), passou a ser composto também pelo próprio hospital psiquiátrico, ofertando dois serviços, quais sejam: leitos em hospital-dia (LHD) e leitos psiquiátricos em hospitais psiquiátricos (LHP). Aqui a contradição apontada pelos críticos da referida Portaria é um suposto abandono do ideário da redução progressiva dos leitos em hospitais psiquiátricos, para um apoio e fomento ao leito hospitalar que ora parecia estar em processo de substituição por rede comunitária, mesmo que de forma não homogênea em todo contexto brasileiro 8 .

Mais polêmico e recente, em fevereiro de 2019, sob o novo governo no Poder Executivo brasileiro, foi lançada a Nota Técnica no 11/2019, intitulada Nova Saúde Mental, publicada pela Coordenação-Geral de Saúde Mental, Álcool e Outras Drogas, Ministério da Saúde 9. Paradigmaticamente ela significa o declínio do caráter "substitutivo" dos serviços de ênfase comunitário e aponta para um "novo" caminho por parte da atual política de saúde nacional. 
Para além da reordenação do caráter "substitutivo" para a ideia de rede "complementar", a política pública em saúde mental passaria a ter também por objetivo, a partir dessa Nota, a ampliação de leitos em hospitais psiquiátricos e comunidades terapêuticas; o financiamento para a compra de aparelhos de eletroconvulsoterapia a serem usados nos serviços hospitalares; e, também, propõe a conversão de serviços "AD" em instituições que poderão praticar a internação de adolescentes como forma de tratamento de dependência química. Tudo isso segue na contramão do disposto nas diretrizes do relatório final da IV Conferência Nacional de Saúde Mental - Intersetorial, realizada em 201010.

Alcançados quase vinte anos desde a homologação da Lei no 10.216/2001, pode-se dizer que o Brasil conta com uma assistência à saúde mental híbrida 11 , composta por serviços de ênfase comunitária e asilares em coexistência, de forma que os serviços asilares são parte da própria rede que inicialmente foi legislada para substituí-los. Tal aspecto parece inviabilizar a identificação do aumento dos serviços da RAPS com o redirecionamento da assistência para um modelo comunitário 12,13 , o que viria materializar o lema da reforma, "por uma sociedade sem manicômios".

Parece-nos, portanto, urgente a necessidade de uma análise crítica da dinâmica de oferta dos serviços no campo da saúde mental, seja para identificar se de fato ocorre a consolidação de uma conquista do movimento social de luta antimanicomial, seja para atender ao proposto pela OMS que, no Atlas de Saúde Mental, de 2017, indica o gerenciamento e a implementação dos serviços de ênfase comunitária como problemática de seus consignatários 14 .

Assim, como parte dos estudos no campo da saúde mental desenvolvidos pelo projeto Coisas Frágeis: Narrativas sobre as Implicações do Diagnóstico Psiquiátrico para a Identidade, o objetivo desta pesquisa consistiu em analisar criticamente a oferta dos serviços de assistência à saúde mental do país. Especificamente, o intuito foi de identificar se ocorreu um processo de substituição dos serviços de ênfase asilar pelos comunitários. Para alcançar tais objetivos, o estudo lançou mão da construção de um Índice de Cobertura Assistencial da Rede de Atenção Psicossocial (iRAPS), como ferramenta para auxílio da análise crítica da reforma psiquiátrica brasileira.

\section{Método}

Trata-se de estudo longitudinal retrospectivo sobre a oferta de serviços de assistência à saúde mental no Brasil, no período de 2008 a 2017, com a elaboração de uma base de dados única. As informações que deram as condições para a construção da base advieram dos dados de acesso público disponibilizados, principalmente, pelo Departamento de Informática do SUS (DATASUS), responsável pelo gerenciamento da oferta dos serviços de saúde no país.

\section{Procedimentos de coleta e análise dos dados}

Utilizando-se a ferramenta TabNet (http://tabnet.datasus.gov.br), foram recolhidas as informações disponíveis do numerário de ofertas dos seguintes serviços/pontos de atenção, em suas diferentes modalidades e pertencentes a distintos componentes da rede, de ênfase comunitária constitutivos da RAPS: Equipe de Consultório de Rua (eCR); Núcleos de Apoio à Saúde da Família (NASF); Centro de Atenção Psicossocial (CAPS); Unidades de Acolhimento (UA); Serviços Residenciais Terapêuticos (SRT); e LHG.

Foram recolhidas informações da oferta de LHP e LHD, os quais são considerados nesta pesquisa como serviços de ênfase asilar, visto que os serviços no período deste estudo eram disponibilizados pelos mesmos hospitais psiquiátricos que prestavam serviços asilares e aos quais inicialmente a RAPS deveria substituir. No caso do serviço LHD, a sua oferta por instituições asilares está em contradição clara à legislação que o constituiu.

Utilizamos dados oriundos do Instituto Brasileiro de Geografia e Estatística (IBGE; https://www. ibge.gov.br) referentes à população dos municípios; por sua vez, no Sistema de Informação em Saúde para a Atenção Básica (SIAB; http://www.datasus.gov.br/SIAB/index.php?area=01) recolhemos o número de Equipes de Saúde da Família (eSF) sobre os municípios analisados.

A compilação do conjunto de dados disponibilizados pelas diferentes bases foi realizada com o auxílio do programa Excel (https://products.office.com/). Dessa forma, construiu-se uma base de 
dados única que detinha informações da oferta dos serviços de assistência à saúde mental, do período histórico de 2008 a 2017, em cada um dos 5.570 municípios do país, constando ainda o seu contingente populacional e o número de eSF disponibilizadas.

O conjunto dessas informações é apresentado em clivagens por região administrativa do país, regiões de saúde e porte da cidade, sendo as duas últimas em função dos parâmetros populacionais estabelecidos para a criação dos CAPS, estratificadas e organizadas nas categorias: pequeno (municípios/regiões de saúde com menos de 20 mil habitantes); médio (20 mil a 70 mil); grande (70.001 a 200 mil); e metrópole (acima de 200 mil) 7.

$\mathrm{Na}$ busca por alcançar os objetivos propostos, além da identificação da oferta desses serviços conforme sua ênfase, seja asilar ou comunitária, foi necessária a identificação da cobertura efetivada por serviços de ênfase comunitária, visto que o aumento do número absoluto de serviços desta ênfase poderia vir a não corresponder à demanda existente.

Cabe ressaltar que cada serviço que atualmente compõe a RAPS foi formalizado legalmente em período distinto e apresenta parametrização específica, que toma como referência desde o número de habitantes da cidade até mesmo a existência de outros serviços, o que torna a análise e apresentação da efetivação de sua cobertura desafiadora. Diante dessas dificuldades, identificamos a cobertura de cada serviço existente no período analisado, conforme legislação vigente, e estabelecemos uma média entre todos os serviços disponibilizados em cada cidade, ponderando a indicação de implementação do serviço no período em questão. Essa média de cobertura do total de serviços de uma cidade, nomeamos como iRAPS, constructo metodológico e instrumental deste estudo.

Observações sobre a criação do iRAPS são necessárias, no que tange a operacionalização dos parâmetros legais de cada serviço. Por exemplo, as legislações referentes às instituições apresentam faixas populacionais específicas para sua implementação. Quando isso ocorreu consideramos, nessas condições, o valor máximo da capacidade de atendimento de cada serviço, preterindo enviesar de forma negativa a análise de cobertura. Assim, explica-se, a seguir, os parâmetros utilizados.

Os parâmetros legais dos serviços CAPS, UA e LHG, conforme suas modalidades, são os seguintes: CAPS I, deve atender cidades com mais de 20 mil habitantes; CAPS II, cidades com até 70 mil; CAPS III, cidades com mais de 200 mil habitantes. Quanto ao recém-instituído CAPS IV, cidades a partir de 500 mil. Porém, quando essa modalidade foi instituída - dezembro de 2017 - o estudo não cobria a sua existência, de modo que não foi incluída no índice; No que diz respeito ao CAPS-i, deve atender municípios com 200 mil habitantes; quanto ao CAPS-AD, apresenta faixa de 70 mil a 300 mil habitantes 15. Já a UA-Adulta (UA-Adu) deve atender áreas com 200 mil habitantes; a UA-Infanto-Juvenil (UA-IJ), 100 mil habitantes 16. Quanto aos LHG, a proporção de 1 para cada 23 mil habitantes 15. O cálculo para a cobertura desses serviços segue a Fórmula 1:

$$
C S x=\frac{N x \times P}{\text { População }} \quad \text { (Fórmula 1) }
$$

onde: CSx é a cobertura do serviço em sua modalidade específica; $N x$, o número de serviços da modalidade ofertada na área; $P$, a população atendida, conforme parâmetro legal do serviço; e População, apopulação da área.

O parâmetro legal estabelecido para a constituição de equipes do NASF está vinculado ao número de eSF ou de populações específicas, de tal forma que a modalidade NASF 1 está vinculada de 5 a 9 equipes, NASF 2 atende de 3 a 4 equipes e o NASF 3 apenas 1 ou 2 equipes 17.

Consideramos para a cobertura do NASF o número de eSF estabelecido por normativa, conforme sua parametrização legal (Fórmula 2), e não o número de eSF existente, posto que apenas 75 cidades no país apresentaram o total adequado ou maior de eSF no ano de 2017. Dessa forma, ao ser considerado o número existente de eSF, nosso estudo apresentaria viés de maior cobertura do NASF devido à quantidade restrita de eSF existentes, ao ser comparada ao seu parâmetro legal.

$$
C b N A S F=\frac{N \times e S F}{e S F i d e a l} \quad \text { (Fórmula 2) }
$$

onde: $C b N A S F$ é a cobertura do serviço em sua modalidade específica; $N$, o número de serviços de cada modalidade ofertada na área; $e S F$, o número de eSF atendida por cada NASF, conforme parâmetro legal do serviço; e eSF ideal, o número de eSF indicado legalmente para ser ofertado na área. 
Os resultantes das Fórmulas 1 e 2 apresentaram valores iniciais de 0. Quando de sua ocorrência indica que a área não é coberta pelo serviço, ao atingir o valor de 1 (inteiro) ou superá-lo, significa que a população da área é atendida com cobertura de 100\% do serviço ou mais (mais assistência do que o previsto por mínimo em normas).

Apesar da amplitude da coleta de informações, alguns componentes da RAPS não foram incluídos no cálculo da iRAPS. Essa opção foi escolhida por três motivos. Primeiro, pelo intuito do estudo em privilegiar o componente comunitário da assistência (ênfase assistencial que seria substitutiva à perspectiva asilar) como meio de evidenciar quantitativamente o movimento da reforma em modo crítico. Segundo, por não garantir-se a fidedignidade do índice no caso deles medirem cobertura de serviços que pertencem majoritariamente a outros campos assistenciais na saúde pública brasileira, como as Unidades Básicas de Saúde (UBS), Serviço de Atendimento Móvel de Urgência (SAMU 192), Sala de Estabilização, Unidade de Pronto Atendimento (UPA 24 horas), empreendimentos solidários e cooperativas sociais, Portas hospitalares de atenção à urgência/pronto socorro em hospital geral, ou seja, a não inclusão justifica-se pelo fato de que os sistemas de informações (SIAB ou DATASUS) que serviram para formular a base de dados não identificam a especificidade da cobertura da assistência à saúde mental destes pontos de atenção/serviços, mesmo que formalmente vinculados à RAPS em distintos componentes. Utilizá-los poderia criar um aumento de cobertura em componentes que ocultariam os hiatos assistenciais quando da falta de serviços que são estratégicos para RAPS (como os CAPS) e também os especializados (como aqueles adotados para compor o iRAPS, neste estudo). E terceiro, devido à legislação de alguns serviços não apresentarem qualquer parâmetro para a sua criação, tornando inviável o cálculo de cobertura destes serviços, que são eles, os Centros de Convivência e Cultura, e o âmbito das iniciativas de trabalho e geração de renda.

Assim, do total das 20 diferentes modalidades de serviços que compõem a RAPS, em seus distintos componentes, todas as 13 modalidades exclusivas ou majoritárias e claramente com agenda de atenção nas práticas de saúde mental compuseram o iRAPS.

O iRAPS, então, permite ao pesquisador, diante de dados sobre quantidades e tipificação de serviço, verificar a efetividade da oferta dos serviços de ênfase comunitária, baseando-se no parâmetro legal estabelecido, e possibilita, por conseguinte, realizar avaliação da assistência em saúde mental do país, no que tange o processo de redirecionamento para uma assistência de ênfase comunitária. Dessa forma, a fórmula para o cálculo do iRAPS, levando-se em consideração os coeficientes de coberturas já descritos, é expressa da seguinte maneira:

$$
i R A P S=\left(\frac{C b N A S F+C b H G+C b U A-I J+C b U A-A d u+C b C A P S-A D+C b C A P S-i+C b C A P S}{N \text { serviços }}\right) \quad \text { (Fórmula 3) }
$$

onde: iRAPS é o índice para avaliação da cobertura do conjunto de serviços da assistência à saúde mental de caráter comunitário; $\mathrm{CbX}$, o valor de cobertura obtido por cada serviço em suas diferentes modalidades; $N$ serviços, o número de serviços em suas diferentes modalidades ofertados na área.

\section{Resultados}

A constituição do banco de dados permitiu expor a primeira evidência deste estudo, que consistiu na identificação do aumento da oferta dos serviços de ênfase comunitária, no período em questão (Tabela 1).

A segunda evidência, possível de obter-se, agora, pela compilação dos serviços de ênfase asilar, consistiu na identificação da redução da oferta LHP de forma não homogênea entre as regiões do país. O serviço de LHP apresentou significativa redução, passando de 51.413 no ano de 2008, para 34.249 leitos no ano de 2017. Verifica-se redução significativa em montante de mais de 12 mil no Sudeste e mais de 5 mil no Nordeste; o Norte apresenta redução de apenas 242 LHP; já o Centro-oeste e o Sul apresentaram aumento de 285 e 77 leitos, respectivamente. Ao considerarmos o porte das cidades, a redução ocorre majoritariamente nas metrópoles e cidades de grande porte.

O serviço de LHD demonstrou um pequeno aumento, com incremento de 141 leitos no decorrer dos dez anos. A oferta desse serviço também tem expressiva variação ao considerarmos as diferentes 
Tabela 1

Quantitativo de serviços de assistência à saúde mental, comunitária e asilar, nos anos 2008 e 2017, por região e país.

\begin{tabular}{|c|c|c|c|c|c|c|c|c|c|c|c|c|c|}
\hline \multirow[t]{2}{*}{ Ênfase } & \multirow[t]{2}{*}{ Serviço } & \multicolumn{2}{|c|}{ Centro-oeste } & \multicolumn{2}{|c|}{ Nordeste } & \multicolumn{2}{|c|}{ Norte } & \multicolumn{2}{|c|}{ Sudeste } & \multicolumn{2}{|c|}{ Sul } & \multicolumn{2}{|c|}{ Brasil } \\
\hline & & 2008 & 2017 & 2008 & 2017 & 2008 & 2017 & 2008 & 2017 & 2008 & 2017 & 2008 & 2017 \\
\hline \multirow[t]{14}{*}{ Comunitária } & CAPS I & 39 & 84 & 272 & 547 & 28 & 103 & 155 & 316 & 126 & 204 & 620 & 1.254 \\
\hline & CAPS II & 22 & 27 & 111 & 148 & 23 & 35 & 156 & 218 & 70 & 85 & 382 & 513 \\
\hline & CAPS III & 0 & 3 & 12 & 24 & 6 & 6 & 51 & 61 & 2 & 9 & 71 & 103 \\
\hline & CAPS-AD & 12 & 21 & 55 & 89 & 4 & 12 & 74 & 153 & 41 & 71 & 186 & 346 \\
\hline & UA-Adu & 0 & 6 & 0 & 40 & 0 & 8 & 0 & 36 & 0 & 30 & 0 & 120 \\
\hline & UA-IJ & 0 & 2 & 0 & 11 & 0 & 1 & 0 & 9 & 0 & 5 & 0 & 28 \\
\hline & eCR I & 0 & 1 & 0 & 5 & 0 & 2 & 0 & 18 & 0 & 9 & 0 & 35 \\
\hline & eCR II & 0 & 3 & 0 & 23 & 0 & 1 & 0 & 30 & 0 & 12 & 0 & 69 \\
\hline & eCR III & 0 & 7 & 0 & 8 & 0 & 4 & 0 & 47 & 0 & 3 & 0 & 69 \\
\hline & SRT I & 0 & 3 & 0 & 44 & 0 & 2 & 0 & 92 & 0 & 11 & 0 & 152 \\
\hline & LHG & 0 & 77 & 0 & 388 & 0 & 79 & 0 & 807 & 0 & 639 & 0 & 1.990 \\
\hline & NASF I & 6 & 150 & 206 & 1.449 & 29 & 249 & 146 & 934 & 37 & 298 & 424 & 3.080 \\
\hline & NASF II & 12 & 91 & 14 & 403 & 1 & 63 & 11 & 267 & 2 & 172 & 40 & 996 \\
\hline & NASF III & 0 & 148 & 0 & 282 & 0 & 97 & 0 & 363 & 0 & 300 & 0 & 1.190 \\
\hline \multirow[t]{2}{*}{ Asilar } & LHP & 2.853 & 3.138 & 11.325 & 6.242 & 620 & 378 & 29.396 & 17.195 & 7.219 & 7.296 & 51.413 & 34.249 \\
\hline & LHD & 342 & 245 & 703 & 816 & 57 & 13 & 964 & 1.097 & 684 & 720 & 2.750 & 2.891 \\
\hline
\end{tabular}

CAPS: Centro de Atenção Psicossocial; CAPS-AD: Centro de Atenção Psicossocial Álcool e Drogas; eCR: Equipe de Consultório na Rua; LHD: Leitos em Hospital-Dia; LHG: Leitos em Hospital Geral; LHP: Leitos Psiquiátricos em Hospitais Psiquiátricos; NASF: Núcleo de Apoio à Saúde da Família; SRT: Serviços Residenciais Terapêuticos; UA-Adu: Unidade de Acolhimento-Adulto; UA-IJ: Unidade de Acolhimento-Infanto-Juvenil.

regiões do país, visto que o Sudeste tem a maior oferta, contando no ano de 2017 com 1.097 LHD, e o Norte, região com menor oferta, com 13 LHD.

Já o incremento do número absoluto de serviços comunitários ficou evidenciado no avanço do iRAPS no decorrer do período analisado, que passou de 0,11 no ano de 2008 e atingiu 0,50 em 2017. Essa progressão confirma a evolução da cobertura dos serviços de ênfase comunitária, em consideração ao nível nacional, e significa que a oferta de serviços de caráter comunitário, em tese, considerando o número de serviços e suas parametrizações legais, efetivaria a cobertura de $50 \%$ da população.

O aumento do iRAPS é verificado, também, ao considerarmos as diferentes regiões administrativas. Porém, tal expansão não ocorre de forma homogênea. A Região Norte apresentou o menor incremento do iRAPS, passando de 0,06 no ano de 2008 para 0,35 em 2017, resultando no avanço de 0,29 do iRAPS. Já à Região Sul do país foi-lhe atribuída o iRAPS de 0,11 no ano de 2008, que no ano de 2017 teve o valor de 0,57, com consequente avanço de 0,45 iRAPS (Tabela 2).

Um fator presente entre todas as regiões é o incremento do iRAPS ser impulsionado pelos resultados das cidades de porte pequeno. Considerando-se as cidades desse porte, verifica-se um aumento do iRAPS expresso em 0,33 na Região Centro-oeste, seguida pelo Norte, com avanço da taxa para 0,37, e do Sudeste com 0,39; tanto as cidades da Região Sul como as do Nordeste atingiram 0,49 o iRAPS. Já as cidades que apresentam o menor incremento do índice são as metrópoles, visto que o avanço mais expressivo no período avaliado é verificado na Região Norte, com índice de 0,08 de incremento do iRAPS; as regiões Centro-oeste e Sul avançaram, respectivamente, 0,04 e 0,03 do iRAPS; já o Nordeste não apresentou variação e o Sudeste reduziu seu iRAPS $(-0,03)$.

Ao se considerar, ainda, o porte das cidades, pode-se verificar que o menor iRAPS no ano de 2017 foi de 0,18 , atribuído às metrópoles da Região Norte. No mesmo ano, as cidades de grande porte da Região Sul atingiram o mais alto iRAPS $(0,77)$, significando que a média de cobertura do conjunto dos serviços de ênfase comunitária nestas cidades é de $77 \%$ da sua população, conforme a parametrização legal.

A análise dos dados quanto à região do país e o porte da cidade permitiu-nos uma percepção da evolução em âmbito nacional, porém, a variação do quantitativo de cidades em cada uma destas cate- 
Tabela 2

Série histórica do Índice de Cobertura da Assistência Psicossocial (iRAPS), de 2008 a 2017, por porte populacional do município, região e país.

\begin{tabular}{|c|c|c|c|c|c|c|c|c|c|c|}
\hline Área & 2008 & 2009 & 2010 & 2011 & 2012 & 2013 & 2014 & 2015 & 2016 & 2017 \\
\hline Brasil & 0,11 & 0,15 & 0,19 & 0,20 & 0,17 & 0,29 & 0,39 & 0,43 & 0,45 & 0,50 \\
\hline Centro-oeste & 0,07 & 0,11 & 0,13 & 0,14 & 0,10 & 0,20 & 0,28 & 0,33 & 0,33 & 0,38 \\
\hline Pequeno & 0,04 & 0,07 & 0,08 & 0,09 & 0,06 & 0,17 & 0,27 & 0,32 & 0,32 & 0,38 \\
\hline Médio & 0,17 & 0,24 & 0,25 & 0,26 & 0,21 & 0,28 & 0,32 & 0,37 & 0,39 & 0,43 \\
\hline Grande & 0,19 & 0,28 & 0,37 & 0,37 & 0,34 & 0,32 & 0,34 & 0,32 & 0,30 & 0,30 \\
\hline Metrópole & 0,22 & 0,24 & 0,27 & 0,27 & 0,21 & 0,26 & 0,26 & 0,27 & 0,27 & 0,26 \\
\hline Nordeste & 0,13 & 0,19 & 0,24 & 0,28 & 0,22 & 0,36 & 0,45 & 0,49 & 0,49 & 0,55 \\
\hline Pequeno & 0,05 & 0,09 & 0,14 & 0,18 & 0,15 & 0,33 & 0,43 & 0,47 & 0,48 & 0,54 \\
\hline Médio & 0,27 & 0,36 & 0,43 & 0,44 & 0,34 & 0,42 & 0,49 & 0,52 & 0,52 & 0,58 \\
\hline Grande & 0,43 & 0,54 & 0,62 & 0,64 & 0,49 & 0,50 & 0,53 & 0,55 & 0,57 & 0,58 \\
\hline Metrópole & 0,33 & 0,35 & 0,39 & 0,39 & 0,26 & 0,27 & 0,30 & 0,33 & 0,33 & 0,33 \\
\hline Norte & 0,06 & 0,12 & 0,16 & 0,19 & 0,14 & 0,22 & 0,29 & 0,31 & 0,32 & 0,35 \\
\hline Pequeno & 0,03 & 0,09 & 0,14 & 0,18 & 0,11 & 0,22 & 0,31 & 0,34 & 0,35 & 0,40 \\
\hline Médio & 0,09 & 0,14 & 0,17 & 0,19 & 0,16 & 0,20 & 0,23 & 0,27 & 0,26 & 0,28 \\
\hline Grande & 0,25 & 0,29 & 0,26 & 0,27 & 0,27 & 0,33 & 0,35 & 0,36 & 0,36 & 0,38 \\
\hline Metrópole & 0,10 & 0,12 & 0,21 & 0,21 & 0,12 & 0,15 & 0,17 & 0,16 & 0,17 & 0,18 \\
\hline Sudeste & 0,11 & 0,14 & 0,17 & 0,19 & 0,16 & 0,21 & 0,31 & 0,37 & 0,41 & 0,47 \\
\hline Pequeno & 0,05 & 0,08 & 0,10 & 0,12 & 0,09 & 0,15 & 0,27 & 0,34 & 0,39 & 0,44 \\
\hline Médio & 0,23 & 0,26 & 0,33 & 0,34 & 0,30 & 0,33 & 0,40 & 0,44 & 0,50 & 0,58 \\
\hline Grande & 0,26 & 0,30 & 0,35 & 0,35 & 0,31 & 0,38 & 0,42 & 0,46 & 0,50 & 0,56 \\
\hline Metrópole & 0,29 & 0,30 & 0,33 & 0,33 & 0,21 & 0,21 & 0,22 & 0,23 & 0,25 & 0,26 \\
\hline Sul & 0,11 & 0,14 & 0,15 & 0,16 & 0,15 & 0,38 & 0,49 & 0,53 & 0,53 & 0,57 \\
\hline Pequeno & 0,06 & 0,08 & 0,08 & 0,08 & 0,09 & 0,33 & 0,46 & 0,52 & 0,51 & 0,55 \\
\hline Médio & 0,27 & 0,31 & 0,36 & 0,36 & 0,33 & 0,55 & 0,59 & 0,59 & 0,58 & 0,62 \\
\hline Grande & 0,42 & 0,51 & 0,66 & 0,64 & 0,53 & 0,60 & 0,65 & 0,74 & 0,74 & 0,77 \\
\hline Metrópole & 0,35 & 0,36 & 0,38 & 0,38 & 0,26 & 0,33 & 0,36 & 0,36 & 0,38 & 0,38 \\
\hline
\end{tabular}

gorias é significativa, visto que o país tem, do seu total, apenas 149 metrópoles e mais de 3.800 municípios de pequeno porte. Para considerar essas especificidades foi identificado o numerário de cidades por porte e, concomitantemente, a estratificação quanto ao iRAPS, obtido da seguinte forma: RAPS Inexistente (cidades de iRAPS 0); RAPS de Baixa Efetividade (iRAPS de 0 a 0,49); RAPS de Média Efetividade (iRAPS de 0,50 a 0,99); RAPS Efetivada (iRAPS a partir de 1). Cinco novos municípios foram criados no período da pesquisa, de maneira que foram considerados sem assistência nos anos que não dispuseram de informações oficiais da população e da oferta de serviços de saúde. Tal estratificação permitiu um novo dimensionamento na evolução do iRAPS, verificado na Tabela 3.

A estratificação com base no nível de cobertura permitiu identificar de imediato que o melhor iRAPS de 2017, referente ao grupo das cidades de grande porte da Região Sul, é composto por 51 municípios. Desses, 22 detêm classificação iRAPS de média efetividade e apenas 12 municípios contavam com completa cobertura, de modo que foram classificados como RAPS Efetivada.

Ainda no ano de 2017, pode-se verificar 439 municípios classificados como RAPS Efetivada, de forma que, destes, 378 são de pequeno ou médio porte. A redução, em todas as regiões, do quantitativo de cidades de pequeno porte na classificação de cobertura RAPS Inexistente e seu incremento com tendência proporcional nas classificações de Baixa e Média cobertura, demonstram nesta clivagem o incremento do iRAPS anteriormente verificado. Mesmo com esse incremento, mais de 70\% das cidades de pequeno porte, em todas as regiões do país, apresentam classificação Inexistente ou Baixa, com exceção do Nordeste que apresenta 57\% dessas cidades nessas classificações. 
Tabela 3

Série histórica do quantitativo de cidades, conforme classificação obtida no iRAPS, porte populacional do município e região do Brasil.

\begin{tabular}{|c|c|c|c|c|c|c|c|c|c|c|c|c|c|c|c|c|c|c|c|c|c|c|c|c|c|}
\hline \multirow{3}{*}{$\begin{array}{l}\text { Região/ } \\
\text { Classifi- } \\
\text { cação }\end{array}$} & \multicolumn{25}{|c|}{ Porte do município } \\
\hline & \multicolumn{5}{|c|}{2013} & \multicolumn{5}{|c|}{2014} & \multicolumn{5}{|c|}{2015} & \multicolumn{5}{|c|}{2016} & \multicolumn{5}{|c|}{2017} \\
\hline & MET & $\mathbf{G}$ & $\mathbf{M}$ & $\mathbf{P}$ & $\mathbf{T}$ & MET & G & M & $\mathbf{P}$ & $\mathbf{T}$ & MET & G & $\mathbf{M}$ & $\mathbf{P}$ & $\mathbf{T}$ & MET & G & $\mathbf{M}$ & $\mathbf{P}$ & $\mathbf{T}$ & MET & $\mathbf{G}$ & $\mathbf{M}$ & $\mathbf{P}$ & $T$ \\
\hline $\begin{array}{l}\text { Centro- } \\
\text {-oeste }\end{array}$ & 9 & 20 & 352 & 86 & 467 & 10 & 19 & 350 & 88 & 467 & 10 & 19 & 348 & 90 & 467 & 10 & 20 & 345 & 92 & 467 & 10 & 20 & 344 & 93 & 467 \\
\hline $\begin{array}{l}\text { Inexis- } \\
\text { tente }\end{array}$ & & 2 & 232 & 20 & 254 & & 2 & 167 & 18 & 187 & & 1 & 131 & 13 & 145 & & 1 & 131 & 11 & 143 & & 1 & 94 & 9 & 104 \\
\hline Baixa & 9 & 13 & 69 & 55 & 146 & 10 & 11 & 108 & 55 & 184 & 10 & 13 & 120 & 60 & 203 & 10 & 14 & 118 & 64 & 206 & 10 & 14 & 136 & 65 & 225 \\
\hline Média & & 3 & 48 & 8 & 59 & & 4 & 69 & 10 & 83 & & 4 & 89 & 12 & 105 & & 4 & 88 & 11 & 103 & & 4 & 106 & 11 & 121 \\
\hline $\begin{array}{l}\text { Efeti- } \\
\text { vada }\end{array}$ & & 2 & 3 & 3 & 8 & & 2 & 6 & 5 & 13 & & 1 & 8 & 5 & 14 & & 1 & 8 & 6 & 15 & & 1 & 8 & 8 & 17 \\
\hline $\begin{array}{l}\text { Nordes- } \\
\text { te }\end{array}$ & 27 & 80 & 1.171 & 516 & 1.794 & 27 & 80 & 1.166 & 521 & 1.794 & 29 & 80 & 1.159 & 526 & 1.794 & 29 & 81 & 1.157 & 527 & 1.794 & 29 & 821 & 1.154 & 5291 & 1.794 \\
\hline $\begin{array}{l}\text { Inexis- } \\
\text { tente }\end{array}$ & & 1 & 463 & 56 & 520 & & & 292 & 42 & 334 & & & 212 & 36 & 248 & & & 209 & 32 & 241 & & & 135 & 16 & 151 \\
\hline Baixa & 25 & 50 & 362 & 326 & 763 & 24 & 48 & 400 & 308 & 780 & 24 & 45 & 421 & 316 & 806 & 24 & 45 & 410 & 316 & 795 & 25 & 46 & 421 & 303 & 795 \\
\hline Média & 2 & 17 & 329 & 102 & 450 & 3 & 19 & 447 & 129 & 598 & 5 & 22 & 487 & 127 & 641 & 5 & 21 & 498 & 133 & 657 & 4 & 20 & 550 & 156 & 730 \\
\hline $\begin{array}{l}\text { Efeti- } \\
\text { vada }\end{array}$ & & 12 & 17 & 32 & 61 & & 13 & 27 & 42 & 82 & & 13 & 39 & 47 & 99 & & 15 & 40 & 46 & 101 & & 16 & 48 & 54 & 118 \\
\hline Norte & 10 & 28 & 271 & 141 & 450 & 10 & 28 & 269 & 143 & 450 & 10 & 29 & 267 & 144 & 450 & 10 & 30 & 264 & 146 & 450 & 11 & 30 & 263 & 146 & 450 \\
\hline $\begin{array}{l}\text { Inexis- } \\
\text { tente }\end{array}$ & & 2 & 160 & 50 & 212 & & 1 & 112 & 41 & 154 & & 1 & 103 & 36 & 140 & & 1 & 102 & 37 & 140 & & 1 & 76 & 28 & 105 \\
\hline Baixa & 10 & 19 & 55 & 80 & 164 & 10 & 21 & 80 & 88 & 199 & 10 & 21 & 79 & 92 & 202 & 10 & 21 & 81 & 94 & 206 & 11 & 20 & 94 & 100 & 225 \\
\hline Média & & 6 & 54 & 8 & 68 & & 5 & 70 & 10 & 85 & & 6 & 77 & 11 & 94 & & 7 & 72 & 10 & 89 & & 8 & 83 & 14 & 105 \\
\hline $\begin{array}{l}\text { Efeti- } \\
\text { vada }\end{array}$ & & 1 & 2 & 3 & 6 & & 1 & 7 & 4 & 12 & & 1 & 8 & 5 & 14 & & 1 & 9 & 5 & 15 & & 1 & 10 & 4 & 15 \\
\hline Sudeste & 72 & 126 & 1.130 & 340 & 1.668 & 72 & 128 & 1.130 & 338 & 1.668 & 72 & 129 & 1.126 & 341 & 1.668 & 73 & 129 & 1.122 & 344 & 1.668 & 73 & 1291 & 1.120 & 3461 & 1.668 \\
\hline $\begin{array}{l}\text { Inexis- } \\
\text { tente }\end{array}$ & 1 & 14 & 857 & 129 & 1001 & 1 & 15 & 641 & 107 & 764 & 1 & 13 & 537 & 97 & 648 & 1 & 11 & 513 & 91 & 616 & 1 & 8 & 417 & 79 & 505 \\
\hline Baixa & 69 & 73 & 140 & 157 & 439 & 69 & 70 & 262 & 162 & 563 & 69 & 68 & 321 & 166 & 624 & 68 & 67 & 317 & 159 & 611 & 67 & 63 & 362 & 158 & 650 \\
\hline Média & 2 & 21 & 106 & 19 & 148 & 2 & 23 & 192 & 32 & 249 & 2 & 24 & 212 & 33 & 271 & 4 & 22 & 227 & 40 & 293 & 5 & 27 & 263 & 41 & 336 \\
\hline $\begin{array}{l}\text { Efeti- } \\
\text { vada }\end{array}$ & & 18 & 27 & 35 & 80 & & 20 & 35 & 37 & 92 & & 24 & 56 & 45 & 125 & & 29 & 65 & 54 & 148 & & 31 & 78 & 68 & 177 \\
\hline Sul & 24 & 51 & 928 & 188 & 1.191 & 26 & 50 & 928 & 187 & 1.191 & 26 & 50 & 925 & 190 & 1.191 & 26 & 51 & 923 & 191 & 1.191 & 26 & 51 & 921 & 1931 & 1.191 \\
\hline $\begin{array}{l}\text { Inexis- } \\
\text { tente }\end{array}$ & 1 & & 731 & 35 & 767 & 1 & & 556 & 26 & 583 & & & 472 & 22 & 494 & & & 461 & 25 & 486 & & & 400 & 18 & 418 \\
\hline Baixa & 20 & 25 & 87 & 101 & 233 & 22 & 24 & 178 & 95 & 319 & 23 & 19 & 214 & 98 & 354 & 22 & 20 & 217 & 96 & 355 & 22 & 17 & 249 & 104 & 392 \\
\hline Média & 3 & 14 & 75 & 19 & 111 & 3 & 16 & 146 & 30 & 195 & 3 & 19 & 179 & 36 & 237 & 4 & 19 & 184 & 39 & 246 & 4 & 22 & 208 & 35 & 269 \\
\hline $\begin{array}{l}\text { Efeti- } \\
\text { vada }\end{array}$ & & 12 & 35 & 33 & 80 & & 10 & 48 & 36 & 94 & & 12 & 60 & 34 & 106 & & 12 & 61 & 31 & 104 & & 12 & 64 & 36 & 112 \\
\hline
\end{tabular}

G: município de grande porte; M: município de médio porte; MET: metrópole; P: município de pequenio porte; T: total de cidades.

Outro fator possível de se verificar é uma tendência em todas as regiões do país de redução do quantitativo de cidades na classificação de Inexistente da RAPS e o concomitante aumento na classificação de Baixa e Média cobertura. Além do fato da classificação cobertura RAPS Efetivada ter apresentado leve incremento.

O quantitativo de cidades conforme o porte também permitiu a análise dos serviços de eCR e SRT que não apresentaram parametrização passível de operacionalização, sendo possível a identificação apenas do contingente ofertado destes serviços (Tabela 4). 
Série histórica do quantitativo de Equipe de Consultório de Rua (eCR) e Serviços Residenciais Terapêuticos (SRT), conforme classificação obtida no Índice de Cobertura da Assistência Psicossocial (iRAPS) e região do Brasil.

\begin{tabular}{|c|c|c|c|c|c|c|c|c|c|c|c|c|c|c|c|c|c|c|}
\hline \multirow{2}{*}{$\begin{array}{l}\text { Região/ } \\
\text { Classifi- } \\
\text { cação }\end{array}$} & \multicolumn{3}{|c|}{2012} & \multicolumn{3}{|c|}{2013} & \multicolumn{3}{|c|}{2014} & \multicolumn{3}{|c|}{2015} & \multicolumn{3}{|c|}{2016} & \multicolumn{3}{|c|}{2017} \\
\hline & eCR & SRT & Cidades & eCR & SRT & Cidades & eCR & SRT & Cidades & eCR & SRT & Cidades & eCR & SRT & Cidades & eCR & SRT & Cidades \\
\hline $\begin{array}{l}\text { Centro- } \\
\text {-oeste }\end{array}$ & 5 & 2 & 467 & 8 & 5 & 467 & 10 & 8 & 467 & 11 & 9 & 467 & 11 & 11 & 467 & 11 & 14 & 467 \\
\hline $\begin{array}{l}\text { Inexis- } \\
\text { tente }\end{array}$ & 0 & 0 & 334 & 0 & 0 & 254 & 0 & 0 & 187 & 0 & 0 & 145 & 0 & 0 & 143 & 0 & 0 & 104 \\
\hline Baixa & 4 & 1 & 117 & 7 & 3 & 146 & 8 & 5 & 184 & 9 & 6 & 203 & 9 & 7 & 206 & 9 & 8 & 225 \\
\hline Média & 1 & 0 & 12 & 1 & 1 & 59 & 2 & 2 & 83 & 2 & 2 & 105 & 2 & 2 & 103 & 2 & 3 & 121 \\
\hline Efetivada & 0 & 1 & 4 & 0 & 1 & 8 & 0 & 1 & 13 & 0 & 1 & 14 & 0 & 2 & 15 & 0 & 3 & 17 \\
\hline Nordeste & 16 & 5 & 1.794 & 21 & 25 & 1.794 & 31 & 34 & 1.794 & 35 & 62 & 1.794 & 36 & 69 & 1.794 & 36 & 73 & 1.794 \\
\hline $\begin{array}{l}\text { Inexis- } \\
\text { tente }\end{array}$ & 0 & 0 & 928 & 0 & 0 & 520 & 0 & 0 & 334 & 0 & 0 & 248 & 0 & 0 & 241 & 0 & 0 & 151 \\
\hline Baixa & 15 & 3 & 615 & 20 & 18 & 763 & 25 & 22 & 780 & 27 & 39 & 806 & 28 & 44 & 795 & 29 & 41 & 795 \\
\hline Média & 1 & 1 & 204 & 1 & 3 & 450 & 6 & 7 & 598 & 8 & 14 & 641 & 8 & 17 & 657 & 7 & 23 & 730 \\
\hline Efetivada & 0 & 1 & 47 & 0 & 4 & 61 & 0 & 5 & 82 & 0 & 9 & 99 & 0 & 8 & 101 & 0 & 9 & 118 \\
\hline Norte & 1 & 1 & 450 & 1 & 0 & 450 & 5 & 0 & 450 & 6 & 1 & 450 & 9 & 3 & 450 & 7 & 4 & 450 \\
\hline $\begin{array}{l}\text { Inexis- } \\
\text { tente }\end{array}$ & 0 & 0 & 289 & 0 & 0 & 212 & 0 & 0 & 154 & 0 & 0 & 140 & 0 & 0 & 140 & 0 & 0 & 105 \\
\hline Baixa & 1 & 1 & 118 & 1 & 0 & 164 & 4 & 0 & 199 & 5 & 1 & 202 & 7 & 3 & 206 & 6 & 4 & 225 \\
\hline Média & 0 & 0 & 38 & 0 & 0 & 68 & 0 & 0 & 85 & 0 & 0 & 94 & 1 & 0 & 89 & 0 & 0 & 105 \\
\hline Efetivada & 0 & 0 & 5 & 0 & 0 & 6 & 1 & 0 & 12 & 1 & 0 & 14 & 1 & 0 & 15 & 1 & 0 & 15 \\
\hline Sudeste & 26 & 44 & 1.668 & 46 & 80 & 1.668 & 66 & 96 & 1.668 & 74 & 165 & 1.668 & 80 & 202 & 1.668 & 95 & 229 & 1.668 \\
\hline $\begin{array}{l}\text { Inexis- } \\
\text { tente }\end{array}$ & 0 & 1 & 1151 & 0 & 1 & 1001 & 0 & 1 & 764 & 0 & 3 & 648 & 0 & 5 & 616 & 0 & 5 & 505 \\
\hline Baixa & 24 & 33 & 367 & 43 & 54 & 439 & 59 & 68 & 563 & 67 & 106 & 624 & 72 & 129 & 611 & 84 & 155 & 650 \\
\hline Média & 1 & 8 & 95 & 2 & 9 & 148 & 4 & 12 & 249 & 4 & 21 & 271 & 5 & 26 & 293 & 8 & 33 & 336 \\
\hline Efetivada & 1 & 2 & 55 & 1 & 16 & 80 & 3 & 15 & 92 & 3 & 35 & 125 & 3 & 42 & 148 & 3 & 36 & 177 \\
\hline Sul & 6 & 1 & 1.191 & 12 & 7 & 1.191 & 14 & 8 & 1.191 & 18 & 13 & 1.191 & 21 & 18 & 1.191 & 24 & 22 & 1.191 \\
\hline $\begin{array}{l}\text { Inexis- } \\
\text { tente }\end{array}$ & 0 & 0 & 921 & 0 & 0 & 767 & 0 & 0 & 583 & 0 & 0 & 494 & 0 & 0 & 486 & 0 & 0 & 418 \\
\hline Baixa & 5 & 1 & 188 & 9 & 5 & 233 & 11 & 6 & 319 & 15 & 8 & 354 & 18 & 13 & 355 & 21 & 16 & 392 \\
\hline Média & 1 & 0 & 52 & 3 & 1 & 111 & 3 & 1 & 195 & 3 & 1 & 237 & 3 & 1 & 246 & 3 & 1 & 269 \\
\hline Efetivada & 0 & 0 & 30 & 0 & 1 & 80 & 0 & 1 & 94 & 0 & 4 & 106 & 0 & 4 & 104 & 0 & 5 & 112 \\
\hline
\end{tabular}

Nota: nesta tabela o período abrangido foi a partir do ano de 2012, devido os dados da oferta de SRT somente estarem disponíveis nesse ano, que também é o ano de criação legal do serviço de eCR.

Foi identificado aumento da oferta de eCR e SRT ao longo do período em análise, seguindo a tendência não homogênea em sua distribuição, conforme ocorreu com os outros serviços que compuseram o iRAPS. Foi possível verificar que os serviços foram criados em um número restrito de municípios, os quais, em sua maioria, apresentaram a classificação de Baixa cobertura.

Considerando-se o grau de cobertura expresso pelo iRAPS, no ano de 2017 o país contabilizou no total 1.283 municípios que receberam classificação de cobertura RAPS Inexistente. São eles, conforme o seu porte populacional: 1.122 pequeno; 150 médio; 10 grande; e 1 metrópole. Desses, 11 municípios de grande porte ou metrópole não dispuseram de qualquer modalidade de serviço que compõe o iRAPS, e apenas 2 municípios pequenos e 3 médios contaram com SRT.

Do total, 2.287 municípios foram classificados como Baixa cobertura da RAPS, que atendem menos do 50\% da população; 1.561 foram classificados com cobertura Média da RAPS; e, por fim, 439 
municípios foram classificados com cobertura Efetivada e dispõem de um conjunto de serviços que presta atendimentos ao total da população.

Antes de passarmos às discussões e análises críticas acerca dos resultados expostos, faz-se necessária a apresentação do iRAPS, considerando então a organização territorial das Regiões de Saúde 18, que agrupa cidades sob o intuito de que trabalhem em conjunto, na oferta de serviços. Assim, quando os parâmetros legais de cada modalidade de serviço tratam de montantes populacionais de municípios, foram considerados os valores correspondentes da Região de Saúde. Seguindo essa especificação, foram organizadas as tais regiões de saúde conforme a região administrativa do país, estratificando-as conforme o iRAPS obtido, e exposto o quantitativo de serviços em cada uma disponível no ano de 2017 (Tabela 5).

Podemos verificar algumas mudanças consideráveis quando comparamos os valores obtidos com a organização territorial em cidades e regiões de saúde. Há redução do iRAPS em todas as regiões administrativas do país, de tal sorte que a Região Centro-oeste passou a apresentar a menor média $(0,22)$ de iRAPS. No outro extremo, a maior média foi obtida pela Região Sudeste, com 0,42. Evidenciou-se, ainda, que nenhuma região extrapolou a média de cobertura de 50\% da população pela RAPS. Ademais, somente uma região de saúde no país recebeu classificação de RAPS Inexistente, em contraponto ao delineamento por municípios, no qual se verifica 1.283 nesta classificação.

Essa clivagem se caracteriza pela concentração de mais de $90 \%$ da população de cada região administrativa do país habitar regiões de saúde que receberam classificação de Baixa efetividade da RAPS, ou seja, que dispõem de serviços de assistência de ênfase comunitária que efetivam cobertura a menos de 50\% dos habitantes. A única exceção é a Região Sul, que apresenta apenas $87 \%$ da população em áreas de Baixa efetividade da RAPS.

Tabela 5

Quantitativo de Regiões de Saúde e serviços de assistência à saúde mental de ênfase comunitária, no ano de 2017, conforme classificação do Índice de Cobertura da Assistência Psicossocial (iRAPS) e região do Brasil.

\begin{tabular}{|c|c|c|c|c|c|c|c|c|c|c|c|c|c|c|c|c|c|c|c|c|}
\hline \multirow{2}{*}{$\begin{array}{l}\text { Região/ } \\
\text { Classificação }\end{array}$} & \multirow[t]{2}{*}{ RS } & \multirow[t]{2}{*}{$\mathbf{P} \%$} & \multicolumn{5}{|c|}{ CAPS } & \multicolumn{2}{|c|}{ UA } & \multicolumn{3}{|c|}{ eCR } & \multicolumn{2}{|c|}{ SRT } & \multirow[t]{2}{*}{ LHG } & \multicolumn{3}{|c|}{ NASF } & \multirow[t]{2}{*}{$\mathbf{Q}$} & \multirow{2}{*}{$\begin{array}{l}\text { Média do } \\
\text { iRAPS }\end{array}$} \\
\hline & & & I & II & III & $\mathbf{i}$ & AD & Adu & IJ & I & II & III & I & II & & I & II & III & & \\
\hline Centro-oeste & 39 & 9,37 & 109 & 34 & 4 & 11 & 25 & 7 & 2 & 1 & 3 & 8 & 3 & 11 & 121 & 181 & 106 & 151 & 6 & 0,22 \\
\hline Baixa & 36 & 98,57 & 106 & 32 & 4 & 10 & 23 & 7 & 2 & 1 & 2 & 8 & 3 & 10 & 121 & 178 & 105 & 141 & 6 & 0,18 \\
\hline Média & 3 & 1,43 & 3 & 2 & 0 & 1 & 2 & 0 & 0 & 0 & 1 & 0 & 0 & 1 & 0 & 3 & 1 & 10 & 5 & 0,67 \\
\hline Norte & 133 & 27,57 & 547 & 148 & 24 & 48 & 89 & 40 & 11 & 5 & 23 & 8 & 44 & 29 & 388 & 1449 & 403 & 282 & 7 & 0,35 \\
\hline Baixa & 111 & 90,95 & 489 & 133 & 14 & 33 & 68 & 27 & 8 & 4 & 22 & 7 & 38 & 27 & 303 & 1290 & 332 & 180 & 7 & 0,27 \\
\hline Média & 18 & 8,39 & 53 & 14 & 9 & 12 & 19 & 12 & 2 & 1 & 1 & 1 & 5 & 2 & 79 & 140 & 61 & 94 & 6 & 0,68 \\
\hline Efetivada & 4 & 0,66 & 5 & 1 & 1 & 3 & 2 & 1 & 1 & 0 & 0 & 0 & 1 & 0 & 6 & 19 & 10 & 8 & 6 & 1,17 \\
\hline Nordeste & 39 & 6,99 & 86 & 30 & 2 & 4 & 9 & 8 & 1 & 2 & 1 & 3 & 2 & 1 & 56 & 210 & 57 & 95 & 6 & 0,20 \\
\hline Inexistente & 1 & 0,34 & 0 & 0 & 0 & 0 & 0 & 0 & 0 & 0 & 0 & 0 & 0 & 0 & 0 & 0 & 0 & 0 & 3 & 0,00 \\
\hline Baixa & 38 & 99,66 & 86 & 30 & 2 & 4 & 9 & 8 & 1 & 2 & 1 & 3 & 2 & 1 & 56 & 210 & 57 & 95 & 6 & 0,21 \\
\hline Sudeste & 149 & 41,80 & 308 & 216 & 64 & 127 & 152 & 35 & 9 & 18 & 30 & 47 & 92 & 138 & 786 & 942 & 258 & 362 & 6 & 0,42 \\
\hline Baixa & 108 & 91,61 & 210 & 182 & 62 & 103 & 114 & 31 & 8 & 18 & 29 & 46 & 80 & 121 & 515 & 788 & 184 & 270 & 6 & 0,24 \\
\hline Média & 31 & 7,19 & 78 & 31 & 2 & 19 & 28 & 1 & 0 & 0 & 1 & 1 & 12 & 15 & 225 & 120 & 57 & 70 & 6 & 0,68 \\
\hline Efetivada & 10 & 1,19 & 20 & 3 & 0 & 5 & 10 & 3 & 1 & 0 & 0 & 0 & 0 & 2 & 46 & 34 & 17 & 22 & 5 & 1,47 \\
\hline Sul & 68 & 14,28 & 204 & 85 & 9 & 46 & 71 & 30 & 5 & 9 & 12 & 3 & 11 & 11 & 639 & 298 & 172 & 300 & 6 & 0,38 \\
\hline Baixa & 54 & 87,08 & 160 & 74 & 8 & 38 & 57 & 22 & 2 & 9 & 9 & 3 & 10 & 7 & 348 & 264 & 152 & 237 & 6 & 0,29 \\
\hline Média & 13 & 12,49 & 42 & 11 & 1 & 8 & 13 & 8 & 3 & 0 & 3 & 0 & 1 & 4 & 278 & 33 & 17 & 62 & 7 & 0,68 \\
\hline Efetivada & 1 & 0,43 & 2 & 0 & 0 & 0 & 1 & 0 & 0 & 0 & 0 & 0 & 0 & 0 & 13 & 1 & 3 & 1 & 5 & 1,08 \\
\hline Brasil & 428 & 100,0 & 1254 & 513 & 103 & 236 & 346 & 120 & 28 & 35 & 69 & 69 & 152 & 190 & 1990 & 3080 & 996 & 1190 & 6 & 0,35 \\
\hline
\end{tabular}

CAPS: Centro de Atenção Psicossocial; CAPS-AD: Centro de Atenção Psicossocial Álcool e Drogas; CAPS-i: Centro de Atenção Psicossocial Infantil; eCR: Equipe de Consultório na Rua; NASF: Núcleo de Apoio à Saúde da Família; P\%: percentual de concentração populacional da área; Q: média de modalidades de serviços disponibilizados na Região de Saúde; RS: Regiões de Saúde; SRT: Serviços Residenciais Terapêuticos; UA-Adu: Unidade de Acolhimento-Adulto; UA-IJ: Unidade de Acolhimento-Infanto-Juvenil. 


\section{Discussão}

O aumento da oferta dos serviços que compõem a RAPS, passando de 891 unidades em suas diferentes modalidades, no ano de 2008, para o numerário de 4.405 serviços ofertados no país em 2017, repercutiu no cálculo do iRAPS no nível nacional, que alcançou o patamar de 0,50. Tal índice significou que a média de cobertura dos serviços de ênfase comunitária no país alcançou $50 \%$ da população no ano de 2017. Aspecto que viria ao encontro da leitura de que o aumento da oferta de serviços indicaria a consolidação da reforma psiquiátrica. Porém, ao iniciarmos o uso do iRAPS, para a análise crítica da oferta dos serviços, já se evidencia uma variação expressiva da cobertura dos serviços entre as regiões administrativas do país, ao verificarmos que o Centro-oeste obteve 0,38 e o Sul 0,57 iRAPS.

A variação identificada no nível regional se perpetua ao considerarmos o porte das cidades. As Metrópoles foram aquelas que apresentam menor iRAPS no ano de 2017, e também não registraram variação do índice no decorrer do período em análise. Tais aspectos apontam para o cenário crítico da assistência nessas cidades, o que é corroborado quando se evidencia que nenhuma cidade desse porte obteve classificação de RAPS Efetivada em 2017. Assim, 90\% do seu numerário obtiveram Baixa cobertura e, ainda, uma delas não apresentou qualquer serviço que compunha o iRAPS. Considerações que são extremamente relevantes devido à concentração de $46 \%$ da população brasileira nas metrópoles.

As cidades de grande porte avançaram 0,24 iRAPS e apresentaram as melhores classificações, visto que 19\% alcançaram a RAPS Efetivada; mais de 75\% apresentaram classificação Média e Baixa efetividade; e apenas $3 \%$ do seu montante foram classificadas em RAPS Inexistente. Porém, as cidades de grande porte são as de menor concentração populacional, com 16\% da população nacional.

As cidades de médio porte, com o aumento de 0,30 de iRAPS, obtiveram o segundo melhor incremento do índice. Mais de 75\% desse grupo registraram classificação de RAPS efetividade Baixa e Média, 13\% RAPS Efetivada e 11\% Inexistente, em 2017. E as cidades médias concentram 22\% da população do país.

As cidades de pequeno porte apresentaram avanço mais expressivo, com incremento médio de 0,44 do iRAPS, e concentravam $16 \%$ da população do país. Esse grupo apresenta distribuição de forma mais homogênea, conforme classificação iRAPS: 30\% Inexistente; 33\% Baixa, 32\% Média e 5\% Efetivada. Fator a ser ressaltado nesse grupo é que esses $5 \%$ de cidades de pequeno porte com classificação Efetivada corresponde a $47 \%$ de todas as 439 cidades que obtiveram a classificação iRAPS Efetivada.

Dessa forma, pode-se inferir, considerando os portes das cidades, que aquelas de grande porte são as com a melhor RAPS, o que se reflete na oferta média de cinco tipos de serviços disponibilizados por elas. Entretanto, essas mesmas cidades concentram o menor porcentual populacional do país; as metrópoles, que concentram quase a metade da população brasileira, não apresentaram avanço da RAPS, no período em estudo, e têm uma média de cobertura abaixo de $50 \%$ da população; as pequenas cidades, que concentram $16 \%$ da população nacional, foram as que mais impulsionaram o avanço do iRAPS em nível nacional, o que se evidencia porque quase a metade das cidades com avaliação RAPS Efetivada é deste grupo.

É válido ressaltar que as cidades de pequeno porte, por contarem com menos de 20 mil habitantes, conforme os parâmetros legais, devem dispor "obrigatoriamente" de apenas dois dos serviços ligados à rede de saúde mental, por nós analisados, o que permite questionar se dois serviços são o suficiente na constituição de uma rede. Além disso, $65 \%$ das cidades de pequeno porte apresentam classificação de RAPS Inexistente ou Baixa, evidenciando que o avanço deste grupo ocorre de forma restrita e que seu impacto no iRAPS nacional ocorre pelo fator quantitativo, posto ser o grupo com a maior quantidade de municípios. Fato também corroborado pelo aumento dos serviços NASF, que parece ser indutor numérico da progressão do índice. Ressaltamos que conforme a regulamentação vigente, a integração de profissionais vinculados diretamente ao âmbito da saúde mental, como psicólogo e médico psiquiátrico, à equipe NASF é opcional, ficando a decisão de sua integração a cargo do gestor 18. Por outro lado, em nosso estudo não consideramos os serviços de eSF e UBS, que apresentam um número significativo nos municípios de pequeno porte e poderia assim influenciar no aumento da cobertura dos serviços de saúde mental.

Enfim, no ano de 2017, 77\% da população brasileira habitaram regiões que ofertavam cobertura menor que 50\% de ênfase comunitária. O avanço mais expressivo da RAPS ocorreu em cidades de 
pequeno porte, que devem contar com até dois tipos de serviços, conforme a legislação. E as metrópoles que concentram quase metade da população nacional não apresentaram melhora. Tais evidências nos autorizam a afirmar que, o aumento da oferta de serviços verificado no período abrangido por este estudo não efetivou a constituição da assistência à saúde mental de caráter comunitário.

Correlacionando, rapidamente, a parametrização legalmente estabelecida que serviu como base para o calculo do iRAPS à cobertura dos serviços expressos pelo iRAPS, podemos apontar que a parametrização legal do CAPS 7 ao ser indicada apenas a municípios com mais de 20 mil habitantes é elevada para a realidade brasileira, seja pelo grande número, 3.802, de municípios não indicados a ofertarem este serviço, seja pelo volume populacional de cada modalidade a ser atendida se distancia da referência da psiquiatria democrática italiana ${ }^{5}$. A parametrização legal da eCR está condicionada ao censo nacional da população em situação de rua, não existente. A legislação do SRT não indica a oferta do serviço em 1.283 municípios, os quais já não dispõem de nenhuma das modalidades de serviço comunitário.

A legislação ainda se apresenta dúbia na regulamentação da UA, que conta com oferta restrita e de baixo impacto no iRAPS, pois se constituir como serviço de ênfase comunitária, no entanto, prevê internamento de até seis meses de duração.

\section{Considerações finais}

Podemos apontar como limitações deste estudo: a não inclusão de determinados serviços, principalmente aqueles da atenção primária, no cálculo iRAPS; a utilização dos dados oriundos do DATASUS, devido estes não serem auditados em sua atualização e os próprios gestores serem responsáveis por sua inclusão, o que possibilita a existência apenas virtual de alguns serviços; e a não análise qualitativa dos serviços disponibilizados.

Todavia, pudemos ainda identificar neste estudo que os desafios à consolidação da RAPS, que ficaram evidentes com o uso do iRAPS indicando a restrita cobertura dos serviços de assistência à saúde mental de caráter comunitário, se não tiveram as suas causas de origem na legislação da reforma, estão fortemente relacionado a ela, visto que se retornarmos ao objetivo da reforma psiquiátrica como a construção de uma sociedade sem manicômios seria esperado que a legislação formalizasse isto. Porém, a Lei no 10.216/2001 indica o redirecionamento ${ }^{3}$, mas não há nenhum documento jurídico estabelecendo metas para a criação de serviços. As parametrizações apresentam-se demasiadamente altas para a realidade brasileira. E por fim, regulamentações mais recentes permitem que hospitais psiquiátricos ofertem serviços comunitários e até mesmo componham a rede com a oferta do tradicional serviço de leito hospitalar que inicialmente buscava-se substituir.

De fato, a problemática legislativa da reforma foi expressa de forma processual. Embrionariamente no Projeto de Lei no 3.657 de 1989, ao se caracterizar como cauteloso e conservador 19, e culminando com a Nota Técnica no 11/2019 que explicitaria, na sua proposição, o fim do redirecionamento comunitário ${ }^{9}$. Assim, esse conjunto de fatores nos permite afirmar que ainda que se perpetue o processo de aumento da oferta dos serviços analisados, conforme os parâmetros estabelecidos, não se trataria da consolidação do objetivo da reforma psiquiátrica, de constituir uma assistência à saúde mental não asilar.

Urge, portanto, a implicação das forças progressistas da sociedade civil no confronto ao atual cenário da política nacional. No âmbito da reforma psiquiátrica, aponta-se a necessidade de coragem teórica, jurídica, ético-política e cultural para realizarmos avaliações profundas sobre as reais conquistas de direitos obtidas no decorrer da História. Colocando-se a questão fulcral: como se poderia considerar a reforma psiquiátrica uma conquista estrutural e sólida se em tão curto período foi negada como possibilidade de negação do lugar da exclusão?

Acreditamos que, prioritariamente, seja necessário o posicionamento de não tomarmos a reforma psiquiátrica como um dogma intocável, mas como uma política em transformação, devemos abrir mão da perspectiva de reparos e remendos da reforma psiquiátrica. Os reparos contraditórios anunciados neste estudo reproduzem o que Boaventura de Souza Santos 20 apontou como a própria ambiguidade da utopia neoliberal: é conservadora porque o que se deve fazer para resolver todos os problemas é radicalizar o presente. $\mathrm{O}$ atual cenário é desafiador, mas será oportuno para reivindicarmos o novo. 


\section{Colaboradores}

C. J. Fernandes, A. F. Lima, P. R. S. Oliveira e W. S. Santos contribuíram na análise e interpretação dos dados, redação do artigo e aprovação final da versão; são responsáveis por todos os aspectos do trabalho e dão garantia da exatidão e integridade de qualquer parte da obra.

\section{Informações adicionais}

ORCID: Cristofthe Jonath Fernandes (0000-00026293-519X); Aluísio Ferreira de Lima (0000-00019747-4701); Pedro Renan Santos de Oliveira (00000001-6308-3227); Walberto Silva dos Santos (00000001-6816-0105).

\section{Referências}

1. Organización Panamericana de la Salud. WHO-AIMS: informe sobre los sistemas de salud mental en América Latina y el Caribe. Washington DC: Organización Panamericana de la Salud; 2013.

2. Caldas de Almeida JM, Horvitz-Lennon M. Mental health care reforms in Latin America: an overview of mental health care reforms in Latin America and the Caribbean. Psychiatr Serv 2010; 61:218-21.

3. Brasil. Lei no 10.216, de 6 de abril de 2001 . Dispõe sobre proteção e direitos das pessoas portadoras de transtornos mentais e redireciona o modelo assistencial em saúde mental. Diário Oficial da União 2001; 9 abr.

4. Advocacia-Geral da União. Caso no 12.237/065 - Damião Ximenes Lopes. http://www.cor teidh.or.cr/docs/casos/ximenes/agesest.pdf (acessado em 12/Mar/2019).

5. Rotelli F, Leonardis O, Mauri D. Desinstitucionalização, uma outra via: a reforma psiquiátrica italiana no contexto da Europa Ocidental e dos "países avançados". In: Nicácio F, organizadora. Desinstitucionalização. São Paulo: Editora Hucitec; 1990. p. 17-59.

6. Ministério da Saúde. Portaria no 3.088, de 23 de dezembro 2011. Institui a Rede de Atenção Psicossocial para pessoas com sofrimento ou transtorno mental e com necessidades decorrentes do uso de crack, álcool e outras drogas, no âmbito do Sistema Único de Saúde (SUS). Diário Oficial da União 2011; 24 dez.

7. Ministério da Saúde. Portaria no 3.588, de 21 de dezembro de 2017. Altera as Portarias Consolidação no 3 e no 6, de 22 dezembro de 2017, para dispor sobre a RAPS, e dá outras providências. Diário Oficial da União 2017; 22 dez.

8. Fernandes CJ, Lima AF, Oliveira PR. Internamento e(sca)ncarado: coexistência da internação psiquiátrica e a rede de atenção psicossocial. Semina: Ciências Sociais e Humanas 2019; 40:25-42.

9. Coordenação-Geral de Saúde Mental, Álcool e Outras Drogas, Departamento de Ações Programáticas Estratégicas, Secretaria de Atenção à Saúde, Ministério da Saúde. Nota Técnica no $11 / 2019$, de 4 de fevereiro de 2019. Esclarecimentos sobre as mudanças na Política Nacional de Saúde Mental e Diretrizes da Política Nacional sobre Drogas. Brasília: Ministério da Saúde; 2019.

10. Conselho Nacional de Saúde. Relatório final da IV Conferência Nacional de Saúde Mental - intersetorial, 27 de junho a 1 de julho de 2010. Brasília: Conselho Nacional de Saúde/ Ministério da Saúde; 2010.

11. Quinderé PHD, Sales FDA, Albuquerque RA, Jorge MSB. A convivência entre os modelos asilar e psicossocial: saúde mental em Fortaleza, CE. Saúde Debate 2010; 34:137-47.

12. Simon AG, Baptista TWF. O papel dos estados na política de saúde mental no Brasil. Cad Saúde Pública 2011; 27:2227-41. 
13. Delfini PSS, Reis AOA. Articulação entre serviços públicos de saúde nos cuidados voltados à saúde mental infantojuvenil. Cad Saúde Pública 2012; 28:357-66.

14. World Health Organization. Mental health atlas 2017. Geneva: World Health Organization; 2018.

15. Ministério da Saúde. Portaria de Consolidação no 3, de 28 de setembro de 2017. Consolidação das normas sobre as redes do Sistema Único de Saúde. Diário Oficial da União 2017; 3 out.

16. Ministério da Saúde. Portaria no 121, de 25 de janeiro de 2012. Institui a Unidade de Acolhimento para pessoas com necessidades decorrentes do uso de Crack, Álcool e Outras Drogas (Unidade de Acolhimento), no componente de atenção residencial de caráter transitório da Rede de Atenção Psicossocial. Diário Oficial da União 2012; 26 jan.

17. Ministério da Saúde. Portaria no 154, de 24 de Janeiro de 2008. Cria os Núcleos de Apoio à Saúde da Família - NASF. Diário Oficial da União 2008; 25 jan.
18. Brasil. Decreto no 7.508 , de 28 de junho de 2011. Regulamenta Lei no 8.080, de 19 de setembro de 1990, para dispor sobre a organização do Sistema Único de Saúde - SUS, o planejamento da saúde, a assistência à saúde e a articulação interfederativa, e dá outras providências. Diário Oficial da União 2011; 29 jun.

19. Câmara dos Deputados. Projeto Lei no 3.657, de 1989. Dispõe sobre a extinção progressiva dos manicômios e sua substituição por outros recursos assistenciais e regulamenta a internação psiquiátrica compulsória. Diário do Congresso Nacional 1989; 29 set.

20. Santos BS. Renovar a teoria crítica e reinventar a emancipação social. São Paulo: Boitempo; 2007. 


\section{Abstract}

Nearly 20 years after the implementation of Brazil's psychiatric reform, the country has a hybrid model of mental health care, in which asylum services are part of the network that should replace them. This study aimed to critically analyze the supply of mental health services in Brazil in order to verify whether there is actually a replacement of asylum-based services by community-based services. A retrospective longitudinal study was performed on the supply of mental health services in Brazil from 2008 to 2017, building a single database on 5,570 municipalities (counties). As an ancillary tool for critical analysis of the psychiatric reform, we developed the Health Coverage Index for the Psychosocial Care Network (iRAPS, in Portuguese), considering the services legal parametrization. The results showed an increase in the supply of community-based services and a reduction in asylum services. However, the iRAPS tool found that $77 \%$ of the Brazilian population lives in areas with low or nonexistent coverage of community services. Small towns, with 16\% of the Brazilian population, were those with the greatest increase in iRAPS. Only 439 cities, $7.9 \%$ of the total, showed total coverage by the Psychosocial Care Network (RAPS, in Portuguese), expressing only $6.69 \%$ of the national population. The metropolises, with $46 \%$ of the Brazilian population, did not show an increase in community services. The analysis of the supply of mental health services based on the iRAPS tool showed that the increase in community services did not occur homogeneously across the country.

Social Psychology; Mental Health; Health Services Coverage; Deinstitutionalization; Psychiatric Hospitals

\section{Resumen}

Transcurridos casi veinte años desde la implantación de la reforma psiquiátrica en Brasil, el país cuenta con una asistencia a la salud mental híbrida, en la que los servicios de acogida a enfermos mentales son parte de la propia red que debería substituirlos. Este estudio buscó analizar críticamente la oferta de los servicios de asistencia a la salud mental del país, con el fin de identificar si, efectivamente, deriva en un proceso de sustitución de los servicios de acogida por parte de los comunitarios. Para alcanzar el objetivo, se realizó un estudio longitudinal retrospectivo de la oferta de servicios de asistencia a la salud mental en Brasil, durante el período de 2008 a 2017, con la elaboración de una base de datos única con información de los 5.570 municipios. Como herramienta auxiliar del análisis-crítico de la reforma psiquiátrica, se creó el Índice de Cobertura Asistencial de la Red de Atención Psicosocial (iRAPS), considerando la parametrización legal de los servicios. Se verificó el avance de la oferta de los servicios comunitarios y la reducción de los de acogida. No obstante, con el iRAPS se identificó que un $77 \%$ de la población brasileña vive en localidades con cobertura de servicios comunitarios baja o inexistente. Las ciudades pequeñas, que concentran a un $16 \%$ de la población nacional, fueron las que más impulsaron el avance del iRAPS a nivel nacional. Apenas 439 ciudades, 7,9\% del total, presentan cobertura asistencial total de la Red de Atención Psicosocial (RAPS), suponiendo solamente un 6,69\% de la poblacional nacional. Las metrópolis, que concentraron un $46 \%$ de la población brasileña, no presentaron un avance en la cobertura de los servicios comunitarios. El análisis de la oferta de servicios de salud mental a partir del iRAPS nos permitió afirmar que el avance de los servicios comunitarios se produjo de forma no homogénea entre las localidades.

Psicología Social; Salud Mental; Cobertura de los Servicios de Salud; Desinstitucionalización; Hospitales Psiquiátricos
Recebido em 12/Mar/2019

Versão final reapresentada em 14/Set/2019

Aprovado em 03/Out/2019 
Fernandes CJ, Lima AF, Oliveira PRS, Santos WS. Índice de Cobertura Assistencial da Rede de Atenção Psicossocial (iRAPS) como ferramenta de análise crítica da reforma psiquiátrica brasileira. Cad Saúde Pública 2020; 36(4):e00049519.

doi: 10.1590/0102-311XER049519

Onde se lê:

Aqui a contradição apontada pelos críticos 8 da referida Portaria é um suposto abandono do ideário da redução progressiva dos leitos em hospitais psiquiátricos, para um apoio e fomento ao leito hospital que ora parecia estar em processo de substituição por rede comunitária.

Leia-se:

Aqui a contradição apontada pelos críticos da referida Portaria é um suposto abandono do ideário da redução progressiva dos leitos em hospitais psiquiátricos, para um apoio e fomento ao leito hospitalar que ora parecia estar em processo de substituição por rede comunitária, mesmo que de forma não homogênea em todo contexto brasileiro 8 .

Onde se lê:

8. Almeida SC. A política antimanicomial brasileira (2008-2013): repensando a implementação e seus condicionantes a partir do nível local [Tese de Doutorado]. Belo Horizonte: Universidade Federal de Minas Gerais; 2015.

Leia-se:

8. Fernandes CJ, Lima AF, Oliveira PR. Internamento e(sca)ncarado: coexistência da internação psiquiátrica e a rede de atenção psicossocial. Semina: Ciências Sociais e Humanas 2019; 40:25-42. 